\title{
MULTIBUMP SOLUTIONS AND ASYMPTOTIC EXPANSIONS FOR MESOSCOPIC ALLEN-CAHN TYPE EQUATIONS*
}

\author{
Matteo Novaga $^{1}$ And EnRICo VAldinoci ${ }^{2}$
}

\begin{abstract}
We consider a mesoscopic model for phase transitions in a periodic medium and we construct multibump solutions. The rational perturbative case is dealt with by explicit asymptotics.
\end{abstract}

Mathematics Subject Classification. 35B40, 49R50.

Received March 25, 2008.

Published online August 20, 2008.

\section{INTRODUCTION}

We are concerned with the equation

$$
-\Delta u+F^{\prime}(u)+H(x)=0, \quad x \in \mathbb{R}^{n},
$$

where the smooth function $F$ is a double-well potential.

More precisely, we assume that

- $F(t) \geqslant 0$ for any $t \in \mathbb{R}$

- $F(t)=0$ if and only if $t= \pm 1$, and $F^{\prime \prime}(1)=F^{\prime \prime}(-1)>0$;

- there exist positive constants $\delta_{0}, c$ such that $F^{\prime}(-1-s) \leqslant-c$ and $F^{\prime}(1+s) \geqslant c$ for any $s \geqslant \delta_{0}$;

- $F(-1+s)=F(1+s)$ for any $s \in\left[-\delta_{0}, \delta_{0}\right]$.

The function $H \in L^{\infty}\left(\mathbb{R}^{n}\right)$ in (1.1) will be a small periodic perturbation of the operator. To this extent, we suppose that

- $\|H\|_{L^{\infty}\left(\mathbb{R}^{n}\right)}$ is suitably small;

- $H$ is $\mathbb{Z}^{n}$-periodic, with zero average on $[0,1]^{n}$, that is

$$
\begin{aligned}
& H(x+k)=H(x) \quad \forall x \in \mathbb{R}^{n} \text { and } k \in \mathbb{Z}^{n} \\
& \text { and } \int_{[0,1]^{n}} H(x) \mathrm{d} x=0 .
\end{aligned}
$$

Keywords and phrases. Oscillatory solutions of PDEs, phase transitions, asymptotic expansions.

* E.V.'s research is partially supported by MURST Variational Methods and Nonlinear Differential Equations.

1 Dipartimento di Matematica, Università di Pisa, Via Buonarroti, 2, 56127 Pisa, Italy. novaga@dm.unipi.it

2 Dipartimento di Matematica, Università di Roma Tor Vergata, Via della Ricerca Scientifica, 1, 00133 Roma, Italy. valdinoci@mat. uniroma2. it 
Equation (1.1) is the Euler-Lagrange equation of the (formal) functional

$$
\int_{\mathbb{R}^{n}} \frac{|\nabla u|^{2}}{2}+F(u)+H(x) u \mathrm{~d} x
$$

The functional in (1.3) has been considered in [10,21] as a mesoscopic model for phase transitions (see also [9] for the analysis of the gradient flow of (1.3), and [8] for a related problem in the random setting).

When $H=0,(1.1)$ is called the Ginzburg-Landau or Allen-Cahn equation, which is a popular model for superconductors and superfluids [15,17] and for gas and solid interfaces [2,25]. Similar equations also arise in cosmology [6].

The term $H$ may be seen as a small defect which favors locally one of the phases: condition (1.2) then says that such defect is "neutral" on large scales, in the sense that both the phases are equally treated.

We refer to [8-10,21] for further physical motivations and geometric interpretations.

In [21], minimizers of (1.3) have been dealt with. We say that $u \in W_{\text {loc }}^{1,2}\left(\mathbb{R}^{n}\right)$ is a minimizer if

$$
\int_{U} \frac{|\nabla u|^{2}}{2}+F(u)+H(x) u \mathrm{~d} x \leqslant \int_{U} \frac{|\nabla(u+\psi)|^{2}}{2}+F(u+\psi)+H(x)(u+\psi) \mathrm{d} x
$$

for any $\psi \in C_{0}^{\infty}(U)$ and any bounded domain $U$ (minimizers of this type are often called "local", or "class A", minimizers). As usual in the calculus of variation framework, the word minimizer for (1.4) refers to the fact that the energy is increased by compact perturbations, even if the energy (1.3) in the whole of $\mathbb{R}^{n}$ may well be infinite.

In particular, the following result has been proved in [21].

Theorem 1.1. For small $\|H\|_{L^{\infty}\left(\mathbb{R}^{n}\right)}$, there exist two $\mathbb{Z}^{n}$-periodic minimizers $U^{ \pm}$of $(1.3)$, with $U^{+}=U^{-}+2$. Also, $U^{+}$and $U^{-}$are uniformly close to +1 and -1 , respectively, in the sense that there exists a constant $C_{0}>0$, independent of $H$, in such a way that

$$
\left\|U^{+}-1\right\|_{L^{\infty}\left(\mathbb{R}^{n}\right)} \leqslant C_{0}\|H\|_{L^{\infty}\left(\mathbb{R}^{n}\right)}, \quad\left\|U^{-}+1\right\|_{L^{\infty}\left(\mathbb{R}^{n}\right)} \leqslant C_{0}\|H\|_{L^{\infty}\left(\mathbb{R}^{n}\right)}
$$

Moreover, given $\omega \in S^{n-1}$, there exist minimizers $u_{\omega}^{ \pm}$of $(1.3)$, which connects $U^{+}$and $U^{-}$far from $\omega^{\perp}$. More explicitly, there are constants $C_{1}, C_{2}>0$, independent of $H$, such that

$$
\left|u_{\omega}^{+}(x)-U^{+}(x)\right|+\left|u_{\omega}^{-}(x)-U^{-}(x)\right| \leqslant C_{1} \mathrm{e}^{-C_{2}\langle\omega, x\rangle}
$$

and

$$
\left|u_{\omega}^{+}(x)-U^{-}(x)\right|+\left|u_{\omega}^{-}(x)-U^{+}(x)\right| \leqslant C_{1} \mathrm{e}^{C_{2}\langle\omega, x\rangle}
$$

for any $x \in \mathbb{R}^{n}$.

The gist of this paper is to detect multibump solutions of the mesoscopic model by gluing together pieces of $u_{\omega}^{ \pm}$'s, according to the following result:

Theorem 1.2. Under a suitable non-degeneracy assumption on $H$ and $\omega \in S^{n-1}$, there exist solutions of (1.1) which connects $U^{+}$and $U^{-}$in the direction given by $\omega$, as many times as we want.

Analogous layered and multibump solutions have been studied in [1,23,24] and multiplicity results are also in [7]: differently from those results, the multibumps are here obtained not by perturbing the potential $F(t)$ into $Q(x) F(t)$, but by using the mesoscopic term $H(x)$. 


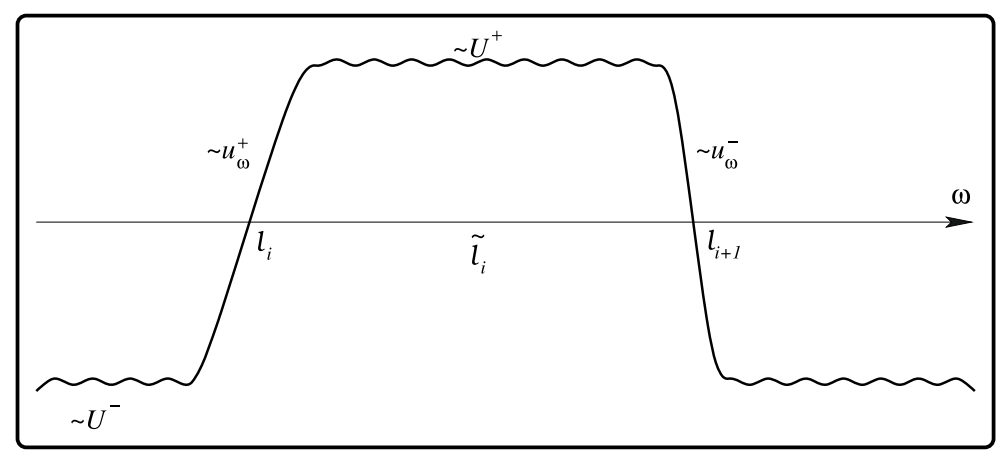

Figure 1. The multibump solution of Theorem 1.3.

A more formal description of Theorem 1.2 will be given in the subsequent Section 2 .

We now state the non-degeneracy condition needed in our paper.

For this, we introduce the following equivalence relation on $\mathbb{R}^{n}$. Given $\omega \in S^{N-1}$ and $x, y \in \mathbb{R}^{n}$, we say that $x \sim_{\omega} y$ if and only if $\langle\omega, x-y\rangle=0$ and $x-y \in \mathbb{Z}^{n}$.

The quotient space $\mathbb{R}^{n} / \sim_{\omega}$ will be denoted by $\mathbb{R}_{\omega}^{n}$.

Let $\omega \in S^{n-1}$ be such that

(A) The minimal eigenvalues $\lambda_{\omega}^{+}$and $\lambda_{\omega}^{-}$of $-\Delta+F^{\prime \prime}\left(u_{\omega}^{ \pm}\right)$in $L^{2}\left(\mathbb{R}_{\omega}^{n}\right)$ are strictly positive and belong to the discrete spectrum of the operator.

Note that condition $(\mathbf{A})$ is an assumption on both $\omega$ and $H$, since $u_{\omega}^{+}$and $u_{\omega}^{-}$depend on $H$ (recall Thm. 1.1). An equivalent formulation of condition $(\mathbf{A})$ is that

$$
\lambda_{\omega}^{ \pm}:=\inf _{\|u\|_{L^{2}\left(\mathbb{R}_{\omega}^{n}\right)}=1} \int_{\mathbb{R}_{\omega}^{n}}|\nabla u|^{2}+F^{\prime \prime}\left(u_{\omega}^{ \pm}\right) u^{2} \mathrm{~d} x
$$

are strictly positive and attained at some eigenfunction $v_{\omega}^{ \pm}$.

Note that, even when (A) fails, the quantity in (1.8) is non-negative, due to the minimizing properties of $u_{\omega}^{ \pm}$ (recall (1.4) and Thm. 1.1).

We reckon that assumption $(\mathbf{A})$ is satisfied for a generic function $H$. Such condition is analogous to the stability condition assumed in [9], and a formal computation is performed in Section 4.2 of [9] to justify such assumption. Related asymptotic expansion of eigenvalues are also in $[4,18]$.

Here, in Section 4, we will make rigorous expansions, interesting in themselves, to make condition $(\mathbf{A})$ more explicit in the rational perturbative case.

The concrete case of small perturbations in a periodic setting will also be considered in Section 4, where we give an explicit, quite general, nondegeneracy condition for the multibump solutions to exist (there, we also relate such condition to a Poincaré-Mel'nikov type non-degeneracy).

Indeed, the following result will be proven in Section 4:

Theorem 1.3. Let $\omega \in \mathbb{Q}^{n}$ and $H(x):=\epsilon h(x)$. Then, the functions $u_{\omega}^{ \pm}$given by Theorem 1.1 approach uniformly, as $\epsilon \rightarrow 0^{+}$, the one-dimensional heteroclinic solutions $\gamma^{ \pm}$of

$$
-\Delta \gamma^{ \pm}+F^{\prime}\left(\gamma^{ \pm}\right)=0
$$

More precisely, for all $\epsilon>0$, there exist $\phi^{ \pm} \in L^{\infty}\left(\mathbb{R}_{\omega}^{n}\right)$ which are solutions of

$$
-\Delta \phi^{ \pm}+F^{\prime \prime}\left(\gamma^{ \pm}\right) \phi^{ \pm}+h=0
$$


in such a way that the functions $u_{\omega}^{ \pm}$have the following asymptotics:

$$
u_{\omega}^{ \pm}(x)=\gamma^{ \pm}(x+o(1) \omega)+\epsilon \phi^{ \pm}(x)+o(\epsilon) .
$$

Moreover, the following eigenvalue expansion hold:

$$
\lambda_{\omega}^{ \pm}=\frac{\epsilon}{\left\|\left(\gamma^{ \pm}\right)^{\prime}\right\|_{L^{2}\left(\mathbb{R}_{\omega}^{n}\right)}^{2}} \int_{\mathbb{R}_{\omega}^{n}} F^{\prime \prime \prime}\left(\gamma^{ \pm}\right)\left(\left(\gamma^{ \pm}\right)^{\prime}\right)^{2} \phi^{ \pm} \mathrm{d} x+o(\epsilon) .
$$

In particular, if $F$ is even

and, if

$$
\lambda_{\omega}^{ \pm}=\frac{\epsilon}{\left\|\left(\gamma^{ \pm}\right)^{\prime}\right\|_{L^{2}\left(\mathbb{R}_{\omega}^{n}\right)}^{2}} \int_{\mathbb{R}_{\omega}^{n}} h(x)\left(\gamma^{ \pm}\right)^{\prime \prime}(x) \mathrm{d} x+o(\epsilon)
$$

$$
\int_{\mathbb{R}_{\omega}^{n}} h(x)\left(\gamma^{ \pm}\right)^{\prime \prime}(x) \mathrm{d} x \neq 0
$$

then the non-degeneracy assumption required in Theorem 1.2 is fulfilled for small $\epsilon$.

In Section 3, we prove Theorem 1.2 (and, in fact, the more explicit version of it given in Thm. 2.1 below), while Section 4 contains comments and examples about the nondegeneracy assumption needed in Theorem 1.2 and an asymptotic expansion for the rational perturbative case, which we think is interesting in itself (see, in particular Thms. 4.1, 4.2 and 4.3 in there).

\section{Formal Setup AND EIGENVALUES}

First we recall an elementary property of the minimal eigenvalue:

Lemma 2.1. Let $f: \mathbb{R}^{n} \rightarrow \mathbb{R}$ be a smooth and $\mathbb{Z}^{n}$-periodic function. Then,

$$
\inf _{\substack{u \in W^{1,2}\left(\mathbb{R}^{n} / \mathbb{Z}^{n}\right) \\\|u\|_{L^{2}\left(\mathbb{R}^{n} / \mathbb{Z}^{n}\right)}=1}} \int_{\mathbb{R}^{n} / \mathbb{Z}^{n}}|\nabla u(x)|^{2}+f(x) u^{2}(x) \mathrm{d} x
$$

is finite and attained at some function $v \in W^{1,2}\left(\mathbb{R}^{n} / \mathbb{Z}^{n}\right)$.

Also, $\{v=0\}=\emptyset$ and, if $\lambda \in \mathbb{R}$ is the quantity in (2.1), we have that

$$
-\Delta v+f v=\lambda v
$$

We omit the standard proof of Lemma 2.1.

We now consider the linearization of (1.1) around a function $u \in L^{\infty}\left(\mathbb{R}^{n}\right)$ :

$$
-\Delta v+F^{\prime \prime}(u) v=\lambda v, \quad \lambda \in \mathbb{R}
$$

and we investigate the properties of its eigenvalues.

Notice that, by Theorem 1.1, we have

$$
F^{\prime \prime}\left(U^{+}\right)=F^{\prime \prime}\left(U^{-}\right)
$$

Then, the following is a plain consequence of Lemma 2.1 and (2.4):

Proposition 2.1. Let $\lambda_{\star}$ be the minimal eigenvalue of the operator $-\Delta+F^{\prime \prime}\left(U^{ \pm}\right)$in $L^{2}\left(\mathbb{R}^{n} / \mathbb{Z}^{n}\right)$. Then $\lambda_{\star}>0$ and there exists a $\mathbb{Z}^{n}$-periodic function $w>0$ satisfying

$$
-\Delta w+F^{\prime \prime}\left(U^{ \pm}\right) w=\lambda_{\star} w
$$

We are now in the position of giving a formal statement of Theorem 1.2, which is the main result of the paper. 
Theorem 2.1. Let $H$ and $\omega \in S^{n-1}$ be such that assumption (A) holds. Then, there exist solutions of (1.1) which connects $U^{+}$and $U^{-}$in the direction given by $\omega$, as many times as we want.

More precisely, there exists a constant $C>0$ such that for any $\epsilon>0$ there exists $\kappa(\epsilon)>0$ with the following property.

Let $N \in \mathbb{Z} \cup\{-\infty\}$ and $M \in \mathbb{Z} \cup\{+\infty\}$, with $N<M$, and $K \geqslant \kappa(\epsilon)$.

Then there exist $\ell_{i} \in \mathbb{R}$, for $i \in \mathbb{Z} \cap[N, M]$, such that, if we set $\tilde{\ell}_{i}:=\left(\ell_{i}+\ell_{i+1}\right) / 2, \ell_{N-1}:=-\infty$ if $N>-\infty$ and $\ell_{M+1}:=+\infty$ if $M<+\infty$, we have:

- $\ell_{j+1}-\ell_{j} \geqslant K$ for any $j \in \mathbb{Z} \cap[N, M-1]$;

- there exists a solution $u$ of (1.1) such that $u(x)$ has distance less than C $\epsilon$ from, alternately, $u_{\omega}^{+}\left(x-\ell_{i} \omega\right)$ and $u_{\omega}^{-}\left(x-\ell_{i} \omega\right)$, for any $x \in \mathbb{R}^{n}$ such that $\langle\omega, x\rangle \in\left(\tilde{\ell}_{i-1}+1, \tilde{\ell}_{i}-1\right)$;

- $u(x)$ has distance less than $C \epsilon$ from, alternately, $U^{+}(x)$ and $U^{-}(x)$ for any $x \in \mathbb{R}^{n}$ such that $\langle\omega, x\rangle \in$ $\left(\tilde{\ell}_{i}-2, \tilde{\ell}_{i}+2\right)$.

In Theorem 2.1 above, we made use of the obvious notation

$$
\begin{gathered}
{[-\infty, a]:=(-\infty, a] \cup\{-\infty\}, \quad[a,+\infty]:=[a,+\infty) \cup\{+\infty\}} \\
\text { and } \quad[-\infty,+\infty]:=\mathbb{R} \cup\{-\infty\} \cup\{+\infty\} .
\end{gathered}
$$

The content of Theorem 2.1 is visualized in Figure 1. Namely, the multibump solution we construct has one and only one excursion from (the vicinity of) one phase to (the vicinity of) the other one in a large interval around $\ell_{i}$, while each of these transitions is suitable glued with the opposite one near $\tilde{\ell}_{i}$.

The gluing near $\tilde{\ell}_{i}$ will be made in order to approximately synchronize $U^{ \pm}\left(x-\omega \ell_{i}\right)$ with $U^{ \pm}\left(x-\omega \ell_{i+1}\right)$ : in fact they will be both almost synchronized with $U^{ \pm}(x)$ (see (3.4) below).

Note that in Theorem 2.1 it is not necessary to require that the size of $H$ is small with respect to $\epsilon$. However, when this happens, any sequence of $\ell_{i}$ 's, that are sufficiently far apart, is favorable to multibumps, provided that condition (A) holds, since the above mentioned synchronization is not needed (namely, (3.4) below will be satisfied just by controlling the oscillations of $U^{ \pm}$by the size of $H$ via Thm. 1.1).

In the perturbative setting, condition (A) may be reduced to a Poincaré-Mel'nikov type non-degeneracy, as discussed in Section 4.

Theorem 2.1 may also be strengthened by taking subsequences of $\ell_{i}$ 's to locate the jumps and by bounding the mutual distance of the $\ell_{i}$ 's from above too. More precisely, the following result also holds:

Theorem 2.2. Let the assumptions of Theorem 2.1 hold.

Then, for any $\epsilon>0$ there exist $\kappa_{+}(\epsilon)>\kappa_{-}(\epsilon)>0$ and a bilateral sequence $\ell:=\left\{\ell_{i}, i \in \mathbb{Z}\right\}$, with $\ell_{i} \in \mathbb{R}$ and $\ell_{i+1}-\ell_{i} \in\left[\kappa_{-}(\epsilon), \kappa_{+}(\epsilon)\right]$, for which given any $\mathcal{J} \subseteq \mathbb{Z}$ and any subsequence $p:=\left\{p_{j}, j \in \mathcal{J}\right\} \subseteq \ell$, there exists a solution $u_{p}$ of $(1.1)$ such that $u_{p}(x)$ has distance less than $\epsilon$ from alternately $u_{\omega}^{+}\left(x-p_{j} \omega\right)$ and $u_{\omega}^{-}\left(x-p_{j} \omega\right)$, if $j \in \mathcal{J}$ and $\langle\omega, x\rangle \in\left(\frac{p_{j-1}+p_{j}}{2}, \frac{p_{j}+p_{j+1}}{2}\right)$.

In Theorem 2.2 we have used again the notation for which $p_{j-1}:=-\infty$ if $j=\inf \mathcal{J}>-\infty$ and $p_{j+1}:=+\infty$ if $j=\sup \mathcal{J}<+\infty$. The proof of Theorem 2.2 is in fact perfectly analogous to the one of Theorem $2.1-$ the reader will just note that the constant $C$ in Theorem 2.1 may be dropped up to relabelling $\epsilon$ and that the synchronization in (3.3), thence the one in (3.4), may be obtained with $\left|\ell_{i}\right| \leqslant \kappa_{*}(\epsilon)$, for some $\kappa_{*}(\epsilon)$, thence $\ell_{i+1}-\ell_{i} \leqslant \kappa_{+}(\epsilon)$.

\section{Proof of Theorem 2.1}

First, let us suppose that $N \neq-\infty$ and $M \neq+\infty$. Up to relabelling $\ell_{i}$, we may suppose that $N=0$, so the points $\ell_{i}$ are just

$$
\ell_{0}, \ell_{1}, \ldots, \ell_{M}
$$


Moreover, given $y \in \mathbb{R}^{n}$, we define

$$
\|y\|_{\mathbb{T}^{n}}:=\inf _{z \in \mathbb{Z}^{n}}|y-z| .
$$

We consider a sequence $\Theta:=\left\{\theta_{j} \in \mathbb{R}, j \in \mathbb{N}\right\}$ for which

$$
\theta_{j+1}-\theta_{j} \geqslant a_{o}, \text { for some } a_{o}>0
$$

and

$$
\lim _{j \rightarrow+\infty}\left\|\omega \theta_{j}\right\|_{\mathbb{T}^{n}}=0
$$

The existence of such a sequence $\Theta$ can be proved by induction over $n$. The inductive step goes as follows. If

$$
\left\{m \in \mathbb{Z}^{n} \text { s.t. } \omega \cdot m=0\right\}=\{0\}
$$

the claim is true (see, e.g., [13], p. 250). If, on the other hand, there exists $m=\left(m^{\prime}, m_{n}\right) \in \mathbb{Z}^{n-1} \times \mathbb{Z}$ with, say, $m_{n}>0$, and such that $\omega \cdot m=0$, we write $\omega=\left(\omega^{\prime}, \omega_{n}\right) \in \mathbb{R}^{n-1} \times \mathbb{R}$ and we apply the inductive hypothesis to $\omega^{\prime}$. This gives the existence of a sequence $s_{j} \in \mathbb{R}$ for which $s_{j+1}-s_{j} \geqslant \alpha_{o}$ for some $\alpha_{o}>0$ and $\left\|\omega^{\prime} s_{j}\right\|_{\mathbb{T}^{n}}$ is infinitesimal. Then, $\theta_{j}:=m_{n} s_{j}$ satisfies (3.1) and (3.2), with $a_{o}:=m_{n} \alpha_{o}$.

In the following, we take the $\ell_{i}$ 's to be far apart elements of the sequence $\Theta$, so that (3.2) implies that

$$
\left\|\omega \ell_{i}\right\|_{\mathbb{T}^{n}} \text { is as small as we wish. }
$$

In particular,

$$
\left|U^{ \pm}\left(x-\omega \ell_{i}\right)-U^{ \pm}(x)\right| \text { is as small as we wish, }
$$

where $U^{ \pm}$is given in Theorem 1.1.

Let $\phi \in C^{\infty}(\mathbb{R},[0,1])$ be such that $\phi(t)=1$ for any $t \geqslant 1$ and $\phi(t)=0$ for any $t \leqslant-1$.

For any $i \in \mathbb{Z} \cap[0, M-1]$, let $\phi_{i}(x):=\phi\left(\langle\omega, x\rangle-\tilde{\ell}_{i}\right)$.

For any $i \in \mathbb{Z} \cap[0, M]$ let also $u_{i}$ be alternately $u_{\omega}^{+}\left(x-\ell_{i} \omega\right)$ and $u_{\omega}^{-}\left(x-\ell_{i} \omega\right)$, as prescribed by Theorem 2.1 .

We also set $v_{i}$ to be either $v_{\omega}^{+}\left(x-\ell_{i} \omega\right)$, if $u_{i}=u_{\omega}^{+}\left(x-\ell_{i} \omega\right)$, or $v_{\omega}^{-}\left(x-\ell_{i} \omega\right)$, if $u_{i}=u_{\omega}^{-}\left(x-\ell_{i} \omega\right)$, where $v_{\omega}^{ \pm}$is given by condition (A), according to (1.8).

The eigenvalue $\lambda_{\omega}^{ \pm}$corresponding to $v_{i}$ will be denoted by $\lambda_{i}$.

Analogously, we set $z_{i}$ (resp., $\hat{z}_{i}$ ) to be either $U^{+}\left(x-\ell_{i} \omega\right)$ (resp., $U^{-}\left(x-\ell_{i} \omega\right)$ ), if $u_{i}=u_{\omega}^{+}\left(x-\ell_{i} \omega\right)$, or $U^{-}\left(x-\ell_{i} \omega\right)$ (resp., $\left.U^{+}\left(x-\ell_{i} \omega\right)\right)$, if $u_{i}=u_{\omega}^{-}\left(x-\ell_{i} \omega\right)$.

Note that, by Theorem 1.1,

$$
\begin{aligned}
& \left|u_{i}(x)-z_{i}(x)\right| \text { gets arbitrarily small for }\langle\omega, x\rangle-\ell_{i} \geqslant R, \text { and } \\
& \left|u_{i}(x)-\hat{z}_{i}(x)\right| \text { gets arbitrarily small for }\langle\omega, x\rangle-\ell_{i} \leqslant-R,
\end{aligned}
$$

provided that $R$ is large enough.

In particular, there exists a suitable $L>0$ in such a way that

$$
\begin{aligned}
& \left|u_{i}(x)-z_{i}(x)\right| \leqslant \frac{\lambda_{\star}}{2\left\|F^{\prime \prime \prime}\right\|_{L^{\infty}([-2,2])}} \text { as long as }\langle\omega, x\rangle-\ell_{i} \geqslant L, \text { and } \\
& \left|u_{i}(x)-\hat{z}_{i}(x)\right| \leqslant \frac{\lambda_{\star}}{2\left\|F^{\prime \prime \prime}\right\|_{L^{\infty}([-2,2])}} \text { as long as }\langle\omega, x\rangle-\ell_{i} \leqslant-L .
\end{aligned}
$$

Recalling (2.4), we also define

Note that

$$
\Phi(x):=F^{\prime \prime}\left(z_{i}(x)\right)=F^{\prime \prime}\left(\hat{z}_{i}(x)\right)
$$

$$
\left|\Phi(x)-F^{\prime \prime}\left(u_{i}(x)\right)\right| \leqslant\left\|F^{\prime \prime \prime}\right\|_{L^{\infty}([-2,2])} \min \left\{\left|u_{i}(x)-z_{i}(x)\right|,\left|u_{i}(x)-\hat{z}_{i}(x)\right|\right\} \quad \text { for any } x \in \mathbb{R}^{n} .
$$


Also,

$$
\sup _{x \in \mathbb{R}^{n}}\left|\Phi(x)-F^{\prime \prime}\left(U^{ \pm}(x)\right)\right|+\left|H(x)-H\left(x-\omega \ell_{i}\right)\right| \text { is as small as we wish, }
$$

due to (3.4) and (3.3).

Given $C>0$, to take suitably large in the sequel, we define

$$
\tilde{u}_{i}^{ \pm}:=u_{i} \pm \epsilon\left(w+C v_{i}\right)
$$

where $w$ is the one of Proposition 2.1.

By (1.8), we know that $\left\|v_{\omega}^{ \pm}\right\|_{L^{2}\left(\mathbb{R}_{\omega}^{n}\right)}=1$ and so

$$
\lim _{R \rightarrow+\infty}\left\|v_{\omega}^{ \pm}\right\|_{L^{2}(|\langle\omega, x\rangle| \geqslant R)}=0
$$

Elliptic regularity [14], Theorem 8.13, then yields

$$
\lim _{R \rightarrow+\infty}\left\|v_{\omega}^{ \pm}\right\|_{C^{2}(|\langle\omega, x\rangle| \geqslant R)}=0
$$

Therefore,

$$
\begin{aligned}
\left\|\tilde{u}_{i+1}^{ \pm}-\tilde{u}_{i}^{ \pm}\right\|_{C^{2}\left(\left|\langle\omega, x\rangle-\tilde{\ell}_{i}\right| \leqslant 2\right)}= & \left\|u_{i+1}-u_{i} \pm C \epsilon\left(v_{i+1}-v_{i}\right)\right\|_{C^{2}\left(\left|\langle\omega, x\rangle-\tilde{\ell}_{i}\right| \leqslant 2\right)} \\
\leqslant & \left\|u_{i+1}-\hat{z}_{i+1}\right\|_{C^{2}\left(\left|\langle\omega, x\rangle-\tilde{\ell}_{i}\right| \leqslant 2\right)}+\left\|u_{i}-z_{i}\right\|_{C^{2}\left(\left|\langle\omega, x\rangle-\tilde{\ell}_{i}\right| \leqslant 2\right)} \\
& +\left\|\hat{z}_{i+1}-z_{i}\right\|_{C^{2}\left(\left|\langle\omega, x\rangle-\tilde{\ell}_{i}\right| \leqslant 2\right)} \\
& +\left\|v_{i+1}\right\|_{C^{2}\left(\left|\langle\omega, x\rangle-\tilde{\ell}_{i}\right| \leqslant 2\right)}+\left\|v_{i}\right\|_{C^{2}\left(\left|\langle\omega, x\rangle-\tilde{\ell}_{i}\right| \leqslant 2\right)} \\
\leqslant & \left\|u_{i+1}-\hat{z}_{i+1}\right\|_{C^{2}\left(\left|\langle\omega, x\rangle-\tilde{\ell}_{i}\right| \leqslant 2\right)}+\left\|u_{i}-z_{i}\right\|_{C^{2}\left(\left|\langle\omega, x\rangle-\tilde{\ell}_{i}\right| \leqslant 2\right)} \\
& +\left\|U^{ \pm}\left(x-\omega \ell_{i+1}\right)-U^{ \pm}\left(x-\omega \ell_{i}\right)\right\|_{C^{2}\left(\left|\langle\omega, x\rangle-\tilde{\ell}_{i}\right| \leqslant 2\right)} \\
& +\left\|v_{\omega}^{ \pm}\right\|_{C^{2}\left(\left|\langle\omega, x\rangle+\ell_{i+1}-\tilde{\ell}_{i}\right| \leqslant 2\right)}+\left\|v_{\omega}^{ \pm}\right\|_{C^{2}\left(\left|\langle\omega, x\rangle+\ell_{i}-\tilde{\ell}_{i}\right| \leqslant 2\right)}
\end{aligned}
$$

is as small as we wish,

if the $\ell_{i}$ 's are far apart, thanks to (3.4), (3.5) and (3.10).

We now define

$$
\begin{aligned}
\beta^{ \pm}(x):= & \left(\prod_{j=0}^{M-1}\left(1-\phi_{j}(x)\right)\right) \tilde{u}_{0}^{ \pm}(x) \\
& +\sum_{i=1}^{M-1}\left(\left(\prod_{j=i}^{M-1}\left(1-\phi_{j}(x)\right)\right) \phi_{i-1}(x) \tilde{u}_{i}^{ \pm}(x)\right) \\
& +\phi_{M-1}(x) \tilde{u}_{M}^{ \pm}(x) .
\end{aligned}
$$

Note that, if $k \in \mathbb{Z} \cap[1, M-1]$ and $\langle\omega, x\rangle \in\left[\tilde{\ell}_{k-1}+1, \tilde{\ell}_{k}-1\right]$, we have that $\phi_{j}(x)=0$ for any $j \geqslant k$ and $\phi_{j}(x)=1$ for any $j \leqslant k-1$, thence

$$
\beta^{ \pm}=\tilde{u}_{k}^{ \pm} \text {if }\langle\omega, x\rangle \in\left[\tilde{\ell}_{k-1}+1, \tilde{\ell}_{k}-1\right]
$$

Also,

$$
\beta^{ \pm}(x)=\tilde{u}_{0}^{ \pm}(x)
$$


if $\langle\omega, x\rangle \leqslant \tilde{\ell}_{0}-1$ and

if $\langle\omega, x\rangle \geqslant \tilde{\ell}_{M}+1$.

$$
\beta^{ \pm}(x)=\tilde{u}_{M}^{ \pm}(x)
$$

Also, if $k \in \mathbb{Z} \cap[0, M-1]$, we have that $\phi_{j}(x)=0$ if $k<j \leqslant M-1$ and $\phi_{j}(x)=1$ if $0 \leqslant j<k$, when $\langle\omega, x\rangle \in\left(\tilde{\ell}_{k}-2, \tilde{\ell}_{k}+2\right)$. Accordingly,

$$
\beta^{ \pm}=\left(1-\phi_{k}\right) \tilde{u}_{k}^{ \pm}+\phi_{k} \tilde{u}_{k+1}^{ \pm} \quad \text { if }\langle\omega, x\rangle \in\left(\tilde{\ell}_{k}-2, \tilde{\ell}_{k}+2\right) .
$$

From (3.15) and (3.11), we deduce that

$$
\left\|\beta^{ \pm}-\tilde{u}_{k}^{ \pm}\right\|_{C^{2}\left(\left\{\langle\omega, x\rangle \in\left(\tilde{\ell}_{k}-2, \tilde{\ell}_{k}+2\right)\right\}\right)} \text { is as small as we like, }
$$

as long as $\ell_{k+1}-\ell_{k}$ is large enough.

As a consequence of (3.12), (3.13), (3.14) and (3.16), we have that for any $x \in \mathbb{R}^{n}$ there exists $i$ in such a way that

$$
\sum_{|j|=0}^{2}\left|D^{j}\left(\beta^{ \pm}-\tilde{u}_{i}^{ \pm}\right)(x)\right| \text { is as small as we like, }
$$

provided that the $\ell_{i}$ 's are conveniently far apart.

We now claim that there exists $c>0$ such that

$$
-\left\|F^{\prime \prime \prime}\right\|_{L^{\infty}([-2,2])} \min \left\{\left|u_{i}-z_{i}\right|,\left|u_{i}-\hat{z}_{i}\right|\right\} w+\lambda_{\star} w+C \lambda_{i} v_{i} \geqslant c
$$

as long as $C$ is chosen suitably large (recall that $w$ and $\lambda_{\star}$ are the ones given by Prop. 2.1).

To prove (3.18), we distinguish two cases. If $\left|\langle\omega, x\rangle-\ell_{i}\right| \geqslant L$, we use (3.6) to get

$$
\begin{aligned}
-\left\|F^{\prime \prime \prime}\right\|_{L^{\infty}([-2,2])} \min \left\{\left|u_{i}-z_{i}\right|,\left|u_{i}-\hat{z}_{i}\right|\right\} w \lambda_{\star} w+C \lambda_{i} v_{i} & \geqslant \frac{\lambda_{\star}}{2} w+C \lambda_{i} v_{i} \\
& \geqslant \frac{\lambda_{\star}}{2} \inf _{\mathbb{R}^{n} / \mathbb{Z}^{n}} w .
\end{aligned}
$$

If, on the other hand, $\left|\langle\omega, x\rangle-\ell_{i}\right| \leqslant L$, we have

$$
-\left\|F^{\prime \prime \prime}\right\|_{L^{\infty}([-2,2])} \min \left\{\left|u_{i}-z_{i}\right|,\left|u_{i}-\hat{z}_{i}\right|\right\} w+\lambda_{\star} w+C \lambda_{i} v_{i} \geqslant-5\left\|F^{\prime \prime \prime}\right\|_{L^{\infty}([-2,2])}+C \lambda_{i} \inf _{|\langle\omega, x\rangle| \leqslant L} v_{i} .
$$

Then, (3.18) follows from (3.19) and (3.20) if $C$ is conveniently large.

Furthermore, recalling the setting of $(3.7)$, we see that

$$
\begin{aligned}
-\Delta \tilde{u}_{i}^{ \pm}+F^{\prime}\left(\tilde{u}_{i}^{ \pm}\right)+H(x)= & -\Delta u_{i}+F^{\prime}\left(u_{i} \pm \epsilon\left(w+C v_{i}\right)\right)+H(x) \pm \epsilon\left(-\Delta w-C \Delta v_{i}\right) \\
= & F^{\prime}\left(u_{i} \pm \epsilon\left(w+C v_{i}\right)\right)-F^{\prime}\left(u_{i}\right) \pm \epsilon\left(\lambda_{\star} w+C \lambda_{i} v_{i}-F^{\prime \prime}\left(U^{ \pm}\right) w-C F^{\prime \prime}\left(u_{i}\right) v_{i}\right) \\
& +H(x)-H\left(x-\ell_{i} \omega\right) \\
= & \pm \epsilon\left(\left(F^{\prime \prime}\left(u_{i}\right)-\Phi\right) w+\lambda_{\star} w+C \lambda_{i} v_{i}\right)+O\left(\epsilon^{2}\right) \\
& +H(x)-H\left(x-\ell_{i} \omega\right) \pm \epsilon\left(\Phi-F^{\prime \prime}\left(U^{ \pm}\right)\right) w
\end{aligned}
$$

As a consequence of the latter estimate, (3.8), (3.9) and (3.18), we deduce that

$$
\begin{aligned}
& -\Delta \tilde{u}_{i}^{+}+F^{\prime}\left(\tilde{u}_{i}^{+}\right)+H(x) \geqslant c \epsilon / 2 \quad \text { and } \\
& -\Delta \tilde{u}_{i}^{-}+F^{\prime}\left(\tilde{u}_{i}^{-}\right)+H(x) \leqslant-c \epsilon / 2 .
\end{aligned}
$$


By (3.17) and (3.21), we gather that

$$
\begin{aligned}
& -\Delta \beta^{+}+F^{\prime}\left(\beta^{+}\right)+H(x) \geqslant c \epsilon / 4 \quad \text { and } \\
& -\Delta \beta^{-}+F^{\prime}\left(\beta^{-}\right)+H(x) \leqslant-c \epsilon / 4
\end{aligned}
$$

as long as $\ell_{i+1}$ and $\ell_{i}$ are all distanced enough (possibly in dependence of $\epsilon$ ).

Let $\eta:=\left(\beta^{+}+\beta^{-}\right) / 2$. Then, $\eta$ is smooth and $\beta^{-}<\eta<\beta^{+}$. Thus, for any $R>0$, we let $u_{R}$ be a solution of

$$
-\Delta u_{R}+F^{\prime}\left(u_{R}\right)+H(x)=0
$$

in the open ball $B_{R}$, with $u=\eta$ on $\partial B_{R}$.

Note that the existence of such $u_{R}$ is warranted, for instance, by direct minimization and that $\beta^{-} \leqslant u_{R} \leqslant \beta^{+}$ by Comparison Principle and (3.22).

Also, by elliptic regularity theory, $u_{R}$ converges, up to subsequences, to some $u$, which is a solution of (1.1) and which is trapped between $\beta^{-}$and $\beta^{+}$.

Such $u$ is the desired multibump solution, thanks to (3.12), (3.13) (3.14), (3.16) and (1.5), thus proving Theorem 2.1 when both $N$ and $M$ are finite.

The case in which $N$ or/and $M$ become infinite is then obtained by taking limits, due to elliptic estimates. This ends the proof of Theorem 2.1.

Remark 3.1. From the above proof it also follows that when $\lambda_{\omega}^{+}>0$ (but possibly $\lambda_{\omega}^{-}=0$ ), then there are homoclinic type connections between $u_{\omega}^{+}\left(x-\ell_{0}\right)$ and $u_{\omega}^{-}\left(x-\ell_{1}\right)$, for $\ell_{1}-\ell_{0}$ suitably large.

Analogously, when $\lambda_{\omega}^{-}>0$ (but possibly $\lambda_{\omega}^{+}=0$ ), then there are homoclinic type connections between $u_{\omega}^{-}\left(x-\ell_{0}\right)$ and $u_{\omega}^{+}\left(x-\ell_{1}\right)$.

That is, if we control only one eigenvalue in (A), we are still able to construct one bump solutions.

\section{ON THE VALIDITY OF THE NON-DEGENERACY ASSUMPTION}

We consider now the case in which $\omega \neq 0$ is rational, i.e, up to normalization, $\omega \in \mathbb{Q}^{n}$. Notice that in this case $\mathbb{R}_{\omega}^{n}$ is the topological product of $\mathbb{R}$ and a $(n-1)$-dimensional torus.

We also suppose that

$$
H_{\epsilon}=\epsilon h,
$$

and we show that, even if assumption (A) is violated for $\epsilon=0$, it does hold, for somewhat generic $h$ 's, if $\epsilon \neq 0$ (see for instance Thm. 4.3 below).

Lemma 4.1. Let $u_{\epsilon}^{ \pm}=u_{\omega}^{ \pm}$be the function given by Theorem 1.1 when $H=\epsilon h$ is as in (4.1). Then, there exists a sequence $\epsilon_{n} \rightarrow 0$ and a smooth function $\gamma^{ \pm}$which is a minimal solution of

$$
-\Delta \gamma^{ \pm}+F^{\prime}\left(\gamma^{ \pm}\right)=0, \quad \text { for any } x \in \mathbb{R}^{n}
$$

satisfying

for suitable $\gamma_{o}^{ \pm}: \mathbb{R} \rightarrow \mathbb{R}$, with

$$
\gamma^{ \pm}(x)=\gamma_{o}^{ \pm}(\langle\omega, x\rangle) \quad \text { for any } x \in \mathbb{R}^{n}
$$

$$
\lim _{t \rightarrow+\infty} \gamma_{o}^{ \pm}(t)= \pm 1, \quad \text { and } \quad \lim _{t \rightarrow-\infty} \gamma_{o}^{ \pm}(t)=\mp 1
$$

for which

uniformly on $\mathbb{R}_{\omega}^{n}$.

$$
\lim _{\epsilon \rightarrow 0} u_{\epsilon}^{ \pm}=\gamma^{ \pm}
$$


Proof. By elliptic regularity estimates and the Ascoli-Arzelà theorem, $u_{\epsilon}^{ \pm}$converges locally uniformly, up to subsequence, to some $\gamma^{ \pm}$. Since $u_{\epsilon}^{ \pm}$is a solution of (1.1) with $H$ as in (4.1), passing to the limit we get (4.2). More precisely, since $u_{\epsilon}^{ \pm}$minimizes the energy (1.4) under compact perturbations with $H$ as in (4.1), passing to the limit we conclude that $\gamma^{ \pm}$minimizes the energy under compact perturbations with $H=0$.

In fact, the limit in (4.5) is uniform, not only locally uniform, in $\mathbb{R}_{\omega}^{n}$. Indeed, suppose, by contradiction, that there exists an infinitesimal sequence $\epsilon_{m}$ and $x_{m} \in \mathbb{R}_{\omega}^{n}$ such that

$$
\left|u_{\epsilon_{m}}^{+}\left(x_{m}\right)-\gamma^{+}\left(x_{m}\right)\right| \geqslant a
$$

for some $a>0$. From (1.6), (1.7) and (1.5),

$$
\left|u_{\epsilon_{m}}^{+}(x)-\gamma^{+}(x)\right| \leqslant C\left(\mathrm{e}^{-C|\langle\omega, x\rangle|}+\epsilon_{m}\right)
$$

and so $\left|\left\langle\omega, x_{m}\right\rangle\right| \leqslant \bar{C}$, for a suitable $\bar{C}>0$, due to (4.6). Then, by the locally uniform convergence,

$$
\left|u_{\epsilon_{m}}^{+}\left(x_{m}\right)-\gamma^{+}\left(x_{m}\right)\right| \leqslant\left\|u_{\epsilon_{m}}^{+}-\gamma^{+}\right\|_{L^{\infty}(\{|\langle\omega, x\rangle| \leqslant \bar{C}\})} \leqslant a / 2
$$

for large $m$, in contradiction with (4.6).

This proves the limit in (4.5) to be uniform in $\mathbb{R}_{\omega}^{n}$.

Accordingly, the limits of $\gamma^{ \pm}$for $\langle\omega, x\rangle \rightarrow \pm \infty$ are uniformly attained, because so are the ones of $u_{\epsilon}^{ \pm}$, in the light of (1.6), (1.7) and (1.5).

Then, the results in the literature on the De Giorgi-Gibbons conjecture (see, e.g., Cor. 7 in [12]) imply the one-dimensional symmetry claimed in (4.3).

From now on, we will fix the sequence $\epsilon_{n}$, which for simplicity we will still call $\epsilon$, and the limit functions $\gamma^{ \pm}$ given by Lemma 4.1 .

Lemma 4.2. The functions $\left(\gamma_{o}^{ \pm}\right)^{\prime}$ are strictly positive on the whole of $\mathbb{R}$.

The standard proof of Lemma 4.2 is omitted.

In what follows, when no confusion is possible, the subindex of $\gamma_{o}^{ \pm}$will be dropped and $\gamma^{ \pm}$will be identified with $\gamma_{o}^{ \pm}$without further comments. In particular, we will denote by $\left(\gamma^{ \pm}\right)^{\prime}$ the derivative of $\gamma^{ \pm}$in the direction given by $\omega$, i.e $\left(\gamma^{ \pm}\right)^{\prime}=\left\langle\nabla \gamma^{ \pm}, \omega\right\rangle=\left(\gamma_{o}^{ \pm}\right)^{\prime}(\langle\omega, x\rangle)$.

We now introduce the Schrödinger operator

$$
T^{ \pm}=-\Delta+F^{\prime \prime}\left(\gamma^{ \pm}(x)\right) .
$$

Lemma 4.3. The spectrum of $T^{ \pm}$is composed of an essential spectrum, corresponding to the unbounded interval $\left[F^{\prime \prime}(1),+\infty\right)$, and of a discrete spectrum, given by a finite number of eigenvalues $0=\lambda_{0}^{ \pm}<\ldots<\lambda_{N}^{ \pm}<F^{\prime \prime}(1)$, with finite multiplicities.

Moreover, the eigenspace corresponding to $\lambda_{0}^{ \pm}=0$ is spanned by the eigenfunction $\left(\gamma^{ \pm}\right)^{\prime} \in L^{2}\left(\mathbb{R}_{\omega}^{n}\right)$.

Proof. The first assertion follows from [16], Theorem 5.7 in Chapter V.5.3.

The fact that $\lambda_{0}^{ \pm}$has multiplicity one follows from the minimality property of $\left(\gamma^{ \pm}\right)^{\prime}$ and the strong maximum principle, applied to the equation $T^{ \pm} v=0$ (indeed, the argument in [11], p. 340, may be repeated verbatim here).

For further spectral results on related equations see [26] and references therein.

We now define

$$
\Im:=\left(\left(\gamma^{ \pm}\right)^{\prime}\right)^{\perp}=\left\{\psi \in L^{2}\left(\mathbb{R}_{\omega}^{n}\right) \text { s.t. } \int_{\mathbb{R}_{\omega}^{n}} \psi\left(\gamma^{ \pm}\right)^{\prime} \mathrm{d} x=0\right\} .
$$

Lemma 4.4. For any $g_{0} \in \Im$ there exists a unique $g_{1} \in \Im$ such that $T^{ \pm} g_{1}=g_{0}$. 
Proof. Notice that $T^{ \pm}$is self-adjoint and its domain is dense in $L^{2}\left(\mathbb{R}_{\omega}^{n}\right)$, thence it is a closed operator, and its image is the orthogonal to the kernel (see, e.g., Sect. II.6 in [5]). Since the kernel of $T^{ \pm}$is spanned by $\left(\gamma^{ \pm}\right)^{\prime}$, due to Lemma 4.3, we get that given any $g_{0} \in \Im$ there exists $\tilde{g}_{1} \in L^{2}\left(\mathbb{R}_{\omega}^{n}\right)$ such that $T^{ \pm} \tilde{g}_{1}=g_{0}$.

We now set

$$
g_{1}:=\tilde{g}_{1}-\frac{\int_{\mathbb{R}_{\omega}^{n}} \tilde{g}_{1}\left(\gamma^{ \pm}\right)^{\prime} \mathrm{d} x}{\left\|\left(\gamma^{ \pm}\right)^{\prime}\right\|_{L^{2}\left(\mathbb{R}_{\omega}^{n}\right)}^{2}}\left(\gamma^{ \pm}\right)^{\prime} .
$$

Such $g_{1}$ lies in $\Im$ and $T^{ \pm} g_{1}=T^{ \pm} \tilde{g}_{1}=g_{0}$.

Moreover, if $T^{ \pm} g_{2}=g_{0}$, with $g_{2} \in \Im$, we have that $T^{ \pm}\left(g_{1}-g_{2}\right)=0$ and so, by Lemma $4.3, g_{1}-g_{2}=C\left(\gamma^{ \pm}\right)^{\prime}$, for some $C \in \mathbb{R}$. Therefore,

$$
C\left\|\left(\gamma^{ \pm}\right)^{\prime}\right\|_{L^{2}\left(\mathbb{R}_{\omega}^{n}\right)}^{2}=\int_{\mathbb{R}_{\omega}^{n}}\left(g_{1}-g_{2}\right)\left(\gamma^{ \pm}\right)^{\prime} \mathrm{d} x=0,
$$

so $C=0$ and $g_{1}=g_{2}$.

In the light of Lemma 4.4, given $g_{0} \in \Im$, we define $\left(T^{ \pm}\right)^{-1} g_{0}$ to be the unique element $g_{1}$ in $\Im$ for which $T^{ \pm} g_{1}=g_{0}$.

Since $T^{ \pm}$is self-adjoint, we have that

$$
\int_{\mathbb{R}_{\omega}^{n}}\left(\left(T^{ \pm}\right)^{-1} f\right) g \mathrm{~d} x=\int_{\mathbb{R}_{\omega}^{n}} f\left(\left(T^{ \pm}\right)^{-1} g\right) \mathrm{d} x,
$$

for any $f, g \in \Im$.

Given $x \in \mathbb{R}_{\omega}^{n}$, we let

$$
\Omega_{x}:=\left\{y \in \mathbb{R}_{\omega}^{n}:\langle\omega, x-y\rangle=0\right\} .
$$

Note that $\Omega_{x}$ is an $(n-1)$-dimensional torus.

Lemma 4.5. Let $x_{0}^{ \pm} \in \mathbb{R}_{\omega}^{n}$ be such that $\gamma^{ \pm}\left(x_{0}^{ \pm}\right)=0$. Then, there exists an infinitesimal sequence $M_{\epsilon}^{ \pm}$for which

$$
\int_{\Omega_{x_{0}^{ \pm}}} u_{\epsilon}^{ \pm}(x) \mathrm{d} x=\gamma^{ \pm}\left(x_{0}^{ \pm}+M_{\epsilon}^{ \pm} \omega\right)\left|\Omega_{x_{0}^{ \pm}}\right| .
$$

Proof. Let

$$
m_{\epsilon}^{ \pm}:=\frac{1}{\left|\Omega_{x_{0}^{ \pm}}\right|} \int_{\Omega_{x_{0}^{ \pm}}} u_{\epsilon}^{ \pm}(x) \mathrm{d} x .
$$

Thanks to (4.5) we get $m_{\epsilon}^{ \pm} \rightarrow 0$, as $\epsilon \rightarrow 0$. By Lemma 4.2, we know that $\gamma_{o}$ is invertible. Thus, the thesis follows by letting $M_{\epsilon}^{ \pm}:=\left(\gamma_{o}^{ \pm}\right)^{-1}\left(m_{\epsilon}^{ \pm}\right)-\left\langle\omega, x_{0}^{ \pm}\right\rangle$.

We will now consider the translated heteroclinic

$$
\gamma_{\epsilon}^{ \pm}(x):=\gamma^{ \pm}\left(x+M_{\epsilon}^{ \pm} \omega\right),
$$

for which there holds

$$
\int_{\Omega_{x_{0}^{ \pm}}} \gamma_{\epsilon}^{ \pm} \mathrm{d} x=\int_{\Omega_{x_{0}^{ \pm}}} u_{\epsilon}^{ \pm} \mathrm{d} x .
$$

We are in the position of improving the asymptotics of Lemma 4.1:

Theorem 4.1. For all $\epsilon>0$, there exist smooth functions $\phi^{ \pm} \in L^{\infty}\left(\mathbb{R}_{\omega}^{n}\right)$ such that

$$
u_{\epsilon}^{ \pm}(x)=\gamma_{\epsilon}^{ \pm}(x)+\epsilon \phi^{ \pm}(x)+o(\epsilon) .
$$

Moreover, $\phi^{ \pm}$are solutions of

$$
-\Delta \phi^{ \pm}+F^{\prime \prime}\left(\gamma^{ \pm}\right) \phi^{ \pm}+h=0 .
$$


Proof. We introduce the cylindrical slab

$$
\mathcal{B}_{R}:=\left\{x \in \mathbb{R}_{\omega}^{n} \text { such that }|\langle\omega, x\rangle| \leqslant R\right\} .
$$

Let

and

$$
\phi_{\epsilon}^{ \pm}:=\frac{u_{\epsilon}^{ \pm}-\gamma_{\epsilon}^{ \pm}}{\epsilon}
$$

$$
c_{\epsilon}^{ \pm}:=\int_{0}^{1} F^{\prime \prime}\left(\gamma_{\epsilon}^{ \pm}+\tau\left(u_{\epsilon}^{ \pm}-\gamma_{\epsilon}^{ \pm}\right)\right) \mathrm{d} \tau .
$$

Note that $c_{\epsilon}^{ \pm}$is a smooth function, which is uniformly bounded in $\epsilon$ and close to $F^{\prime \prime}\left(\gamma^{ \pm}\right)$for small $\epsilon$, by (4.5), and that

$$
L_{\epsilon}^{ \pm} \phi_{\epsilon}^{ \pm}+h=0,
$$

where we defined the operator

$$
L_{\epsilon}^{ \pm}:=-\Delta+c_{\epsilon}^{ \pm}
$$

We claim that, for any $R \geqslant 1$ there exists $C_{R}>0$, independent of $\epsilon$, such that

$$
\left\|\phi_{\epsilon}^{ \pm}\right\|_{L^{2}\left(\mathcal{B}_{R}\right)} \leqslant C_{R}
$$

For this, we denote by $U_{\epsilon}^{ \pm}=U^{ \pm}$the $\mathbb{Z}^{n}$-periodic minimizers of Theorem 1.1 and we consider the functions

$$
\psi_{\epsilon}^{ \pm}:=\frac{U_{\epsilon}^{ \pm} \mp 1}{\epsilon},
$$

which solve the equation

$$
-\Delta \psi_{\epsilon}^{ \pm}+d_{\epsilon}^{ \pm} \psi_{\epsilon}^{ \pm}+h=0
$$

where

$$
d_{\epsilon}^{ \pm}:=\int_{0}^{1} F^{\prime \prime}\left( \pm 1+\tau\left(U_{\epsilon}^{ \pm} \mp 1\right)\right) \mathrm{d} \tau
$$

Recall that, from (1.5),

$$
\left\|\psi_{\epsilon}^{ \pm}\right\|_{L^{\infty}\left(\mathbb{R}_{\omega}^{n}\right)} \leqslant C\|h\|_{L^{\infty}\left(\mathbb{R}_{\omega}^{n}\right)}
$$

where the constant $C$ does not depend on $\epsilon$.

We now let

$$
\eta_{\epsilon}^{ \pm}:=\phi_{\epsilon}^{ \pm}-\psi_{\epsilon}^{ \pm} .
$$

From Theorem 1.1, we have that the functions $\eta_{\epsilon}^{ \pm}$lie in $W^{2,2}\left(\mathbb{R}_{\omega}^{n}\right)$, and solve

$$
L_{\epsilon}^{ \pm} \eta_{\epsilon}^{ \pm}=\left(d_{\epsilon}^{ \pm}-c_{\epsilon}^{ \pm}\right) \psi_{\epsilon}^{ \pm} .
$$

Notice that, since $u_{\epsilon}^{ \pm}$converge exponentially to $U_{\epsilon}^{ \pm}$independently of $\epsilon$, we have

$$
\left\|d_{\epsilon}^{ \pm}-c_{\epsilon}^{ \pm}\right\|_{L^{2}\left(\mathbb{R}_{\omega}^{n}\right)} \leqslant C,
$$

for some constant $C>0$ independent of $\epsilon$.

Let now $\mu_{\epsilon}^{ \pm}$be the minimal eigenvalue of the operator $L_{\epsilon}^{ \pm}$on $L^{2}\left(\mathbb{R}_{\omega}^{n}\right)$, and $w_{\epsilon}^{ \pm}>0$ the corresponding eigenvector, which we may take with $L^{2}\left(\mathbb{R}_{\omega}^{n}\right)$-norm equal to 1 . Notice that, as $\epsilon \rightarrow 0$, we have that $\mu_{\epsilon}^{ \pm}$is simple, $\mu_{\epsilon}^{ \pm} \rightarrow 0$ and $w_{\epsilon}^{ \pm} \rightarrow \pm\left(\gamma^{ \pm}\right)^{\prime} /\left\|\left(\gamma^{ \pm}\right)^{\prime}\right\|_{L^{2}\left(\mathbb{R}_{\omega}^{n}\right)}$, uniformly on compact subsets of $\mathbb{R}_{\omega}^{n}$, due to Lemma 4.3, the continuity properties of the eigenvalues [16], Chapter IV.3.5, and the regularity estimates for $w_{\epsilon}^{ \pm}$[14], Theorem 8.13 . 
In particular, by Lemma 4.2 there exists $c>0$ such that

$$
w_{\epsilon}^{ \pm}(y) \geqslant c \quad \text { for all } y \in \Omega_{x_{0}^{ \pm}} .
$$

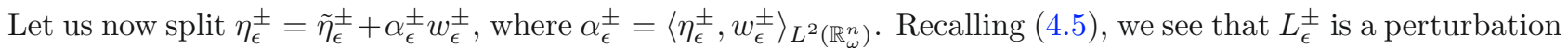
of $T^{ \pm}$and so, by Lemma 4.3 and [16], p. 208, Theorem 3.1, we see that $\left[-\sigma_{o}, \sigma_{o}\right]$ does not meet the spectrum of $L_{\epsilon}^{ \pm}$except that in $\mu_{\epsilon}^{ \pm}$, for some suitably small $\sigma_{o}>0$, independent of $\epsilon$.

As a consequence, we get

$$
\int_{\mathbb{R}_{\omega}^{n}}\left(L_{\epsilon}^{ \pm} \eta_{\epsilon}^{ \pm}\right) \tilde{\eta}_{\epsilon}^{ \pm} \mathrm{d} x=\int_{\mathbb{R}_{\omega}^{n}}\left(L_{\epsilon}^{ \pm} \tilde{\eta}_{\epsilon}^{ \pm}\right) \tilde{\eta}_{\epsilon}^{ \pm} \mathrm{d} x \geqslant \sigma_{o}\left\|\tilde{\eta}_{\epsilon}^{ \pm}\right\|_{L^{2}\left(\mathbb{R}_{\omega}^{n}\right)}^{2}
$$

and so, recalling (4.15), (4.16) and (4.14), we get

$$
\begin{aligned}
\left\|\tilde{\eta}_{\epsilon}^{ \pm}\right\|_{L^{2}\left(\mathbb{R}_{\omega}^{n}\right)} & \leqslant \frac{C}{\left\|\tilde{\eta}_{\epsilon}^{ \pm}\right\|_{L^{2}\left(\mathbb{R}_{\omega}^{n}\right)}} \int_{\mathbb{R}_{\omega}^{n}}\left(L_{\epsilon}^{ \pm} \eta_{\epsilon}^{ \pm}\right) \tilde{\eta}_{\epsilon}^{ \pm} \mathrm{d} x \\
& \leqslant C\left\|d_{\epsilon}^{ \pm}-c_{\epsilon}^{ \pm}\right\|_{L^{2}\left(\mathbb{R}_{\omega}^{n}\right)}\left\|\psi_{\epsilon}^{ \pm}\right\|_{L^{\infty}\left(\mathbb{R}_{\omega}^{n}\right)} \\
& \leqslant C\|h\|_{L^{\infty}\left(\mathbb{R}_{\omega}^{n}\right)} .
\end{aligned}
$$

Since, by (4.15),

$$
\begin{aligned}
\alpha_{\epsilon}^{ \pm} L_{\epsilon}^{ \pm} w_{\epsilon}^{ \pm} & =\left\langle\eta_{\epsilon}^{ \pm}, w_{\epsilon}^{ \pm}\right\rangle_{L^{2}\left(\mathbb{R}_{\omega}^{n}\right)} \mu_{\epsilon}^{ \pm} w_{\epsilon}^{ \pm} \\
& =\left\langle\eta_{\epsilon}^{ \pm}, L_{\epsilon}^{ \pm} w_{\epsilon}^{ \pm}\right\rangle_{L^{2}\left(\mathbb{R}_{\omega}^{n}\right)} w_{\epsilon}^{ \pm} \\
& =\left\langle L_{\epsilon}^{ \pm} \eta_{\epsilon}^{ \pm}, w_{\epsilon}^{ \pm}\right\rangle_{L^{2}\left(\mathbb{R}_{\omega}^{n}\right)} w_{\epsilon}^{ \pm} \\
& =\left\langle\left(d_{\epsilon}^{ \pm}-c_{\epsilon}^{ \pm}\right) \psi_{\epsilon}^{ \pm}, w_{\epsilon}^{ \pm}\right\rangle_{L^{2}\left(\mathbb{R}_{\omega}^{n}\right)} w_{\epsilon}^{ \pm}
\end{aligned}
$$

we see that $\tilde{\eta}_{\epsilon}^{ \pm}$solves the equation

$$
L_{\epsilon}^{ \pm} \tilde{\eta}_{\epsilon}^{ \pm}=\left(d_{\epsilon}^{ \pm}-c_{\epsilon}^{ \pm}\right) \psi_{\epsilon}^{ \pm}-\left\langle\left(d_{\epsilon}^{ \pm}-c_{\epsilon}^{ \pm}\right) \psi_{\epsilon}^{ \pm}, w_{\epsilon}^{ \pm}\right\rangle_{L^{2}\left(\mathbb{R}_{\omega}^{n}\right)} w_{\epsilon}^{ \pm} .
$$

Therefore, recalling (4.14), (4.16) and (4.18), elliptic regularity [14], Theorem 8.12, yields

$$
\begin{aligned}
\left\|\tilde{\eta}_{\epsilon}^{ \pm}\right\|_{W^{2,2}\left(\mathbb{R}_{\omega}^{n}\right)} & \leqslant C\left(\left\|\tilde{\eta}_{\epsilon}^{ \pm}\right\|_{L^{2}\left(\mathbb{R}_{\omega}^{n}\right)}+\left\|\left(d_{\epsilon}^{ \pm}-c_{\epsilon}^{ \pm}\right) \psi_{\epsilon}^{ \pm}\right\|_{L^{2}\left(\mathbb{R}_{\omega}^{n}\right)}\right) \\
& \leqslant C\|h\|_{L^{\infty}\left(\mathbb{R}_{\omega}^{n}\right)} .
\end{aligned}
$$

We let $\bar{\eta}_{\epsilon}^{ \pm}: \mathbb{R} \rightarrow \mathbb{R}$ be the average of $\tilde{\eta}_{\epsilon}^{ \pm}$on sections of $\mathbb{R}_{\omega}^{n}$ orthogonal to $\omega$, i.e

$$
\bar{\eta}_{\epsilon}^{ \pm}(t):=\frac{1}{\left|\Omega_{t \omega}\right|} \int_{\Omega_{t \omega}} \tilde{\eta}_{\epsilon}^{ \pm} \mathrm{d} x .
$$

From (4.19) and the one-dimensional Sobolev Embedding theorem [5], Theorem IX.12, we get

$$
\left\|\bar{\eta}_{\epsilon}^{ \pm}\right\|_{L^{\infty}(\mathbb{R})} \leqslant C\left\|\bar{\eta}_{\epsilon}^{ \pm}\right\|_{W^{2,2}(\mathbb{R})} \leqslant C\left\|\tilde{\eta}_{\epsilon}^{ \pm}\right\|_{W^{2,2}\left(\mathbb{R}_{\omega}^{n}\right)} \leqslant C\|h\|_{L^{\infty}\left(\mathbb{R}_{\omega}^{n}\right)} .
$$

In order to obtain (4.13), it remains to bound the coefficient $\alpha_{\epsilon}^{ \pm}$. Recalling (4.8) and (4.14), we have

$$
\frac{1}{\left|\Omega_{x_{0}^{ \pm}}\right|}\left|\int_{\Omega_{x_{0}^{ \pm}}} \eta_{\epsilon}^{ \pm} \mathrm{d} x\right| \leqslant C\|h\|_{L^{\infty}\left(\mathbb{R}_{\omega}^{n}\right)} .
$$


Therefore, by (4.17) and (4.20),

$$
\begin{aligned}
c\left|\alpha_{\epsilon}^{ \pm}\right| & \leqslant \frac{\left|\alpha_{\epsilon}^{ \pm}\right|}{\left|\Omega_{x_{0}^{ \pm}}\right|}\left|\int_{\Omega_{x_{0}^{ \pm}}} w_{\epsilon}^{ \pm} \mathrm{d} x\right| \\
& =\frac{1}{\left|\Omega_{x_{0}^{ \pm}}\right|}\left|\int_{\Omega_{x_{0}^{ \pm}}}\left(\eta_{\epsilon}^{ \pm}-\tilde{\eta}_{\epsilon}^{ \pm}\right) \mathrm{d} x\right| \leqslant C\|h\|_{L^{\infty}\left(\mathbb{R}_{\omega}^{n}\right)} .
\end{aligned}
$$

This estimate, together with (4.14) and (4.18), gives (4.13).

It follows from (4.12), (4.13) and standard elliptic estimates (see, e.g., [11], Sect. 6.3.1) that $\phi_{\epsilon}^{ \pm}$converges, up to subsequence, to some $\phi^{ \pm} \in L^{\infty}\left(\mathbb{R}_{\omega}^{n}\right)$, uniformly on compact subsets of $\mathbb{R}_{\omega}^{n}$. Hence, (4.9) is a consequence of (4.11).

Passing to the limit in (4.12) and recalling Lemma 4.1, we finally obtain (4.10).

Proposition 4.1. Let

$$
\lambda_{\epsilon}^{ \pm}:=\inf _{\|u\|_{L^{2}\left(\mathbb{R}_{\omega}^{n}\right)}=1} \int_{\mathbb{R}_{\omega}^{n}}|\nabla u|^{2}+F^{\prime \prime}\left(u_{\epsilon}^{ \pm}\right) u^{2} \mathrm{~d} x .
$$

Then, $\lambda_{\epsilon}^{ \pm}$belongs to the discrete spectrum of the operator and

$$
\lambda_{\epsilon}^{ \pm}=\frac{\epsilon}{\left\|\left(\gamma^{ \pm}\right)^{\prime}\right\|_{L^{2}\left(\mathbb{R}_{\omega}^{n}\right)}^{2}} \int_{\mathbb{R}_{\omega}^{n}} F^{\prime \prime \prime}\left(\gamma^{ \pm}\right)\left(\left(\gamma^{ \pm}\right)^{\prime}\right)^{2} \phi^{ \pm} \mathrm{d} x+o(\epsilon)
$$

Proof. Since, by (4.9),

$$
\int_{\mathbb{R}_{\omega}^{n}}|\nabla u|^{2}+F^{\prime \prime}\left(u_{\epsilon}^{ \pm}\right) u^{2} \mathrm{~d} x \leqslant \int_{\mathbb{R}_{\omega}^{n}}|\nabla u|^{2}+F^{\prime \prime}\left(\gamma_{\epsilon}^{ \pm}\right) u^{2} \mathrm{~d} x+\epsilon\left\|F^{\prime \prime \prime}\right\|_{L^{\infty}([-2,2])}\left\|\phi^{ \pm}\right\|_{L^{\infty}\left(\mathbb{R}_{\omega}^{n}\right)} \int_{\mathbb{R}_{\omega}^{n}} u^{2} \mathrm{~d} x
$$

we get

$$
\lambda_{\epsilon}^{ \pm} \leqslant C \epsilon .
$$

Since $\lambda_{\epsilon}^{ \pm}$is small, according to Lemma 4.3 and the continuity properties of the spectrum (see [16], Chap. IV), it does not lie in the essential spectrum of $-\Delta+F^{\prime \prime}\left(u_{\epsilon}^{ \pm}\right)$, hence it belongs to the discrete spectrum.

Let now $w_{\epsilon}^{ \pm}$be the eigenvector corresponding to $\lambda_{\epsilon}^{ \pm}$such that

$$
\left\|w_{\epsilon}^{ \pm}\right\|_{L^{2}\left(\mathbb{R}_{\omega}^{n}\right)}=1
$$

i.e. there holds

$$
\lambda_{\epsilon}^{ \pm}=\int_{\mathbb{R}_{\omega}^{n}}\left|\nabla w_{\epsilon}^{ \pm}\right|^{2}+F^{\prime \prime}\left(u_{\epsilon}^{ \pm}\right)\left(w_{\epsilon}^{ \pm}\right)^{2} \mathrm{~d} x
$$

Then, by (4.9),

$$
\lambda_{\epsilon}^{ \pm}=\int_{\mathbb{R}_{\omega}^{n}}\left|\nabla w_{\epsilon}^{ \pm}\right|^{2}+F^{\prime \prime}\left(\gamma_{\epsilon}^{ \pm}\right)\left(w_{\epsilon}^{ \pm}\right)^{2}+\epsilon F^{\prime \prime \prime}\left(\gamma_{\epsilon}^{ \pm}\right)\left(w_{\epsilon}^{ \pm}\right)^{2} \phi^{ \pm} \mathrm{d} x+o(\epsilon) .
$$

In particular, $\left\|\nabla w_{\epsilon}^{ \pm}\right\|_{L^{2}\left(\mathbb{R}_{\omega}^{n}\right)}$ is uniformly bounded, thence we may suppose that

$$
w_{\epsilon}^{ \pm} \text {converges to some } w^{ \pm} \text {weakly in } W^{1,2}\left(\mathbb{R}_{\omega}^{n}\right) \text { and strongly in } L_{\text {loc }}^{2}\left(\mathbb{R}_{\omega}^{n}\right) .
$$

Recall that from Lemma 4.3 and the spectral theorem we have

$$
\int_{\mathbb{R}_{\omega}^{n}}|\nabla u|^{2}+F^{\prime \prime}\left(\gamma_{\epsilon}^{ \pm}\right) u^{2} \mathrm{~d} x \geqslant \lambda_{\star}^{ \pm} \int_{\mathbb{R}_{\omega}^{n}} \hat{u}^{2} \mathrm{~d} x
$$


where $\lambda_{\star}^{ \pm}>0$ (here we set $\lambda_{\star}^{ \pm}=F^{\prime \prime}(1)$ if 0 is the only discrete eigenvalue),

$$
\kappa:=1 /\left\|\left(\gamma^{ \pm}\right)^{\prime}\right\|_{L^{2}\left(\mathbb{R}^{n}\right)} \text { and } \hat{u}:=u-\kappa^{2}\left\langle u,\left(\gamma_{\epsilon}^{ \pm}\right)^{\prime}\right\rangle_{L^{2}\left(\mathbb{R}_{\omega}^{n}\right)}\left(\gamma_{\epsilon}^{ \pm}\right)^{\prime} .
$$

Since

$$
\lim _{\epsilon \rightarrow 0} \int_{\mathbb{R}_{\omega}^{n}}\left|\nabla w_{\epsilon}^{ \pm}\right|^{2}+F^{\prime \prime}\left(\gamma_{\epsilon}^{ \pm}\right)\left(w_{\epsilon}^{ \pm}\right)^{2} \mathrm{~d} x=0,
$$

due to (4.22) and (4.25), it follows from (4.27) that

$$
\left\|\widehat{w}_{\epsilon}^{ \pm}\right\|_{L^{2}\left(\mathbb{R}_{\omega}^{n}\right)}^{2} \leqslant C \epsilon .
$$

As a consequence, recalling also (4.23) and (4.26), we conclude that

$$
w_{\epsilon}^{ \pm} \text {converges to } w^{ \pm}:=\kappa\left(\gamma^{ \pm}\right)^{\prime} \text { in } L^{2}\left(\mathbb{R}_{\omega}^{n}\right) \text {, as } \epsilon \rightarrow 0 .
$$

Moreover, since $\widehat{w}_{\epsilon}^{ \pm}$solves the equation

$$
T_{\epsilon}^{ \pm} \widehat{w}_{\epsilon}^{ \pm}:=-\Delta \widehat{w}_{\epsilon}^{ \pm}+F^{\prime \prime}\left(\gamma_{\epsilon}^{ \pm}\right) \widehat{w}_{\epsilon}^{ \pm}=\lambda_{\epsilon}^{ \pm} \widehat{w}_{\epsilon}^{ \pm}+\left(F^{\prime \prime}\left(\gamma_{\epsilon}^{ \pm}\right)-F^{\prime \prime}\left(u_{\epsilon}^{ \pm}\right)\right) w_{\epsilon}^{ \pm},
$$

by elliptic regularity [14], Corollary 8.7, and recalling Theorem 4.1, (4.22) and (4.28) we get

$$
\begin{aligned}
\left\|\widehat{w}_{\epsilon}^{ \pm}\right\|_{W^{1,2}\left(\mathbb{R}_{\omega}^{n}\right)}^{2} & \leqslant C\left(\left\|\lambda_{\epsilon}^{ \pm} \widehat{w}_{\epsilon}^{ \pm}\right\|_{L^{2}\left(\mathbb{R}_{\omega}^{n}\right)}^{2}+\left\|\left(F^{\prime \prime}\left(\gamma_{\epsilon}^{ \pm}\right)-F^{\prime \prime}\left(u_{\epsilon}^{ \pm}\right)\right) w_{\epsilon}^{ \pm}\right\|_{L^{2}\left(\mathbb{R}_{\omega}^{n}\right)}^{2}\right) \\
& \leqslant C\left\|u_{\epsilon}^{ \pm}-\gamma_{\epsilon}^{ \pm}\right\|_{L^{2}\left(\mathbb{R}_{\omega}^{n}\right)}^{2}+o(\epsilon)=o(\epsilon) .
\end{aligned}
$$

In particular, it follows that

$$
\int_{\mathbb{R}_{\omega}^{n}} T_{\epsilon}^{ \pm} w_{\epsilon}^{ \pm} w_{\epsilon}^{ \pm} \mathrm{d} x=\int_{\mathbb{R}_{\omega}^{n}} T_{\epsilon}^{ \pm} \widehat{w}_{\epsilon}^{ \pm} \widehat{w}_{\epsilon}^{ \pm} \mathrm{d} x=\int_{\mathbb{R}_{\omega}^{n}}\left|\nabla w_{\epsilon}^{ \pm}\right|^{2}+F^{\prime \prime}\left(\gamma_{\epsilon}^{ \pm}\right)\left(w_{\epsilon}^{ \pm}\right)^{2} \mathrm{~d} x=o(\epsilon) .
$$

Accordingly, exploiting (4.25), (4.29) and (4.30), we get

$$
\begin{aligned}
\lambda_{\epsilon}^{ \pm}+o(\epsilon) & =\int_{\mathbb{R}_{\omega}^{n}}\left|\nabla w_{\epsilon}^{ \pm}\right|^{2}+F^{\prime \prime}\left(\gamma_{\epsilon}^{ \pm}\right)\left(w_{\epsilon}^{ \pm}\right)^{2}+\epsilon F^{\prime \prime \prime}\left(\gamma_{\epsilon}^{ \pm}\right)\left(w_{\epsilon}^{ \pm}\right)^{2} \phi^{ \pm} \mathrm{d} x \\
& =\int_{\mathbb{R}_{\omega}^{n}} T_{\epsilon}^{ \pm} w_{\epsilon}^{ \pm} w_{\epsilon}^{ \pm} \mathrm{d} x+\epsilon \int_{\mathbb{R}_{\omega}^{n}} F^{\prime \prime \prime}\left(\gamma_{\epsilon}^{ \pm}\right)\left(w_{\epsilon}^{ \pm}\right)^{2} \phi^{ \pm} \mathrm{d} x \\
& =\epsilon \kappa^{2} \int_{\mathbb{R}_{\omega}^{n}} F^{\prime \prime \prime}\left(\gamma^{ \pm}\right)\left(\left(\gamma^{ \pm}\right)^{\prime}\right)^{2} \phi^{ \pm} \mathrm{d} x+o(\epsilon) .
\end{aligned}
$$

This proves (4.21).

Lemma 4.6. We have that

$$
\int_{\mathbb{R}_{\omega}^{n}} h\left(\gamma^{ \pm}\right)^{\prime} \mathrm{d} x=0
$$

Proof. From Theorem 4.1,

$$
\begin{aligned}
-\int_{\mathbb{R}_{\omega}^{n}} h\left(\gamma^{ \pm}\right)^{\prime} \mathrm{d} x & =\int_{\mathbb{R}_{\omega}^{n}}-\Delta \phi^{ \pm}\left(\gamma^{ \pm}\right)^{\prime}+F^{\prime \prime}\left(\gamma^{ \pm}\right) \phi^{ \pm}\left(\gamma^{ \pm}\right)^{\prime} \mathrm{d} x \\
& =\int_{\mathbb{R}_{\omega}^{n}}\left(-\left(\gamma^{ \pm}\right)^{\prime \prime \prime}+F^{\prime \prime}\left(\gamma^{ \pm}\right)\left(\gamma^{ \pm}\right)^{\prime}\right) \phi^{ \pm} \mathrm{d} x=0,
\end{aligned}
$$

as desired. 
Notice that condition (4.31) identifies $\gamma^{ \pm}$, which is determined up to a translation along $\omega$, in dependence of the function $h$.

Lemma 4.7. Let $f \in \Im$, and assume that $f$ decays exponentially, possibly with its derivatives, in the directions given by $\pm \omega$. Then, $v^{ \pm}:=\left(T^{ \pm}\right)^{-1} f \in \Im$ enjoys the same decay properties of $f$, and

$$
\int_{\mathbb{R}_{\omega}^{n}} f \phi^{ \pm} \mathrm{d} x=-\int_{\mathbb{R}_{\omega}^{n}} v^{ \pm} h \mathrm{~d} x
$$

Proof. We first observe that, thanks to Lemma 4.4, there exists a unique $v^{ \pm} \in \Im$ such that $T^{ \pm} v^{ \pm}=f$. The decay properties of $v^{ \pm}$then follow from the decay properties of $f$ by elliptic regularity [14], Theorem 8.13. In particular, $v^{ \pm} \in L^{1}\left(\mathbb{R}_{\omega}^{n}\right)$ so that the right-hand side of (4.32) makes sense.

Since, by (4.10), $T^{ \pm} \phi^{ \pm}=-h$ and $T^{ \pm}$is self-adjoint on $L^{2}\left(\mathbb{R}_{\omega}^{n}\right)$, (4.32) can now be easily obtained by approximating $\phi^{ \pm}$with functions $\phi_{R}^{ \pm}:=\phi^{ \pm} \rho_{R}$, where $\rho_{R}$ are suitable cut-off functions with support in $\mathcal{B}_{R}$.

Theorem 4.2. Suppose that $F$ is even. Then,

$$
\lambda_{\epsilon}^{ \pm}=\frac{\epsilon}{\left\|\left(\gamma^{ \pm}\right)^{\prime}\right\|_{L^{2}\left(\mathbb{R}_{\omega}^{n}\right)}^{2}} \int_{\mathbb{R}_{\omega}^{n}} h(x)\left(\gamma^{ \pm}\right)^{\prime \prime}(x) \mathrm{d} x+o(\epsilon) .
$$

Proof. Since $F$ is even, we have that $\gamma_{o}^{ \pm}\left(\cdot+\left\langle\omega, x_{0}^{ \pm}\right\rangle\right)$is odd, and so

$$
\int_{\mathbb{R}_{\omega}^{n}} F^{\prime \prime \prime}\left(\gamma^{ \pm}\right)\left(\left(\gamma^{ \pm}\right)^{\prime}\right)^{2}\left(\gamma^{ \pm}\right)^{\prime} \mathrm{d} x=0
$$

so that we can apply Lemma 4.7 with $f=F^{\prime \prime \prime}\left(\gamma^{ \pm}\right)\left(\left(\gamma^{ \pm}\right)^{\prime}\right)^{2}$.

Then, from (4.32) we get

$$
\int_{\mathbb{R}_{\omega}^{n}} F^{\prime \prime \prime}\left(\gamma^{ \pm}\right)\left(\left(\gamma^{ \pm}\right)^{\prime}\right)^{2} \phi^{ \pm} \mathrm{d} x=-\int_{\mathbb{R}_{\omega}^{n}}\left(T^{ \pm}\right)^{-1}\left(F^{\prime \prime \prime}\left(\gamma^{ \pm}\right)\left(\left(\gamma^{ \pm}\right)^{\prime}\right)^{2}\right) h \mathrm{~d} x .
$$

Hence, by (4.21) we have

$$
\begin{aligned}
\lambda_{\epsilon}^{ \pm} & =\frac{\epsilon}{\left\|\left(\gamma^{ \pm}\right)^{\prime}\right\|_{L^{2}\left(\mathbb{R}_{\omega}^{n}\right)}^{2}} \int_{\mathbb{R}_{\omega}^{n}} F^{\prime \prime \prime}\left(\gamma^{ \pm}\right)\left(\left(\gamma^{ \pm}\right)^{\prime}\right)^{2} \phi^{ \pm} \mathrm{d} x+o(\epsilon) \\
& =-\frac{\epsilon}{\left\|\left(\gamma^{ \pm}\right)^{\prime}\right\|_{L^{2}\left(\mathbb{R}_{\omega}^{n}\right)}^{2}} \int_{\mathbb{R}_{\omega}^{n}}\left(T^{ \pm}\right)^{-1}\left(F^{\prime \prime \prime}\left(\gamma^{ \pm}\right)\left(\left(\gamma^{ \pm}\right)^{\prime}\right)^{2}\right) h \mathrm{~d} x+o(\epsilon) .
\end{aligned}
$$

We also observe that, as a consequence of (4.2),

$$
\begin{aligned}
T^{ \pm}\left(\left(\gamma^{ \pm}\right)^{\prime \prime}\right) & =T^{ \pm}\left(F^{\prime}\left(\gamma^{ \pm}\right)\right) \\
& =-\Delta\left(F^{\prime}\left(\gamma^{ \pm}\right)\right)+F^{\prime \prime}\left(\gamma^{ \pm}\right) F^{\prime}\left(\gamma^{ \pm}\right) \\
& =-F^{\prime \prime \prime}\left(\gamma^{ \pm}\right)\left(\left(\gamma^{ \pm}\right)^{\prime}\right)^{2}-F^{\prime \prime}\left(\gamma^{ \pm}\right)\left(\gamma^{ \pm}\right)^{\prime \prime}+F^{\prime \prime}\left(\gamma^{ \pm}\right) F^{\prime}\left(\gamma^{ \pm}\right) \\
& =-F^{\prime \prime \prime}\left(\gamma^{ \pm}\right)\left(\left(\gamma^{ \pm}\right)^{\prime}\right)^{2} .
\end{aligned}
$$

This and (4.35) imply the desired claim.

We are now in the position to give explicit conditions that imply $(\mathbf{A})$ in the rational perturbative setting, when the potential $F$ is even. 
For this, we also recall that

$$
\lambda_{\epsilon}^{ \pm} \geqslant 0
$$

due to the minimality of $u_{\epsilon}^{ \pm}$.

Proposition 4.2. Let $F$ be an even function, and suppose that $h$ satisfies

$$
\int_{\mathbb{R}_{\omega}^{n}} h(x)\left(\gamma^{ \pm}\right)^{\prime \prime}(x) \mathrm{d} x \neq 0 .
$$

Then, condition (A) is fulfilled by $H=\epsilon h$, for $\epsilon$ small enough.

Proof. By Theorem 4.2 and (4.37), we have that $\lambda_{\epsilon}^{ \pm} \neq 0$, for $\epsilon$ small enough. In fact, from (4.36), we necessarily have that $\lambda_{\epsilon}^{ \pm}>0$, for $\epsilon$ small enough.

Thus, $\lambda_{\epsilon}^{ \pm}$is strictly positive, and lies in the discrete spectrum of the operator by Proposition 4.1.

We now better clarify (4.37). Note that $\gamma^{+}$and $\gamma^{-}$are determined by $h$ itself, in the sense that $h$ selects the translation of $\gamma_{o}^{ \pm}$from which $u_{\epsilon}^{ \pm}$bifurcates. This selection occurs due to (4.31) and to the minimality of $u_{\epsilon}^{ \pm}$.

We introduce the notation

$$
f_{t}(x):=f(x+\omega t)
$$

for a given function $f$ and $t \in \mathbb{R}$.

We observe that, if $F$ is even, the two heteroclinic orbits $\gamma_{o}^{+}$and $\gamma_{o}^{-}$are the same up to sign-change and translation, that is we can write $\gamma^{+}=\gamma_{\theta^{+}}$and $\gamma^{-}=-\gamma_{\theta^{-}}$for a suitable heteroclinic $\gamma$ and suitable $\theta^{ \pm} \in \mathbb{R}$.

We consider the function

$$
\mathbb{R} \ni t \longmapsto \mathcal{F}(t):=\int_{\mathbb{R}_{\omega}^{n}} h(x) \gamma_{t}^{\prime}(x) \mathrm{d} x .
$$

The function $\mathcal{F}$ is periodic since $h$ is periodic and $\omega$ is rational. Also, condition (4.31) says that

$$
\mathcal{F}\left(\theta^{+}\right)=0=\mathcal{F}\left(\theta^{-}\right)
$$

In this spirit, we now prove that condition $(\mathbf{A})$ is assured if these zeroes are non-degenerate:

Theorem 4.3. Let $F$ be even and suppose that

$$
\{\mathcal{F}=0\} \cap\left\{\mathcal{F}^{\prime}=0\right\}=\emptyset \text {. }
$$

Then, condition (A) holds true for $H=\epsilon h$, and $\epsilon$ small enough.

Proof. By (4.39) and (4.40),

$$
0 \neq \mathcal{F}^{\prime}\left(\theta^{ \pm}\right)=\int_{\mathbb{R}_{\omega}^{n}} h \gamma_{\theta^{ \pm}}^{\prime \prime} \mathrm{d} x= \pm \int_{\mathbb{R}_{\omega}^{n}} h\left(\gamma^{ \pm}\right)^{\prime \prime} \mathrm{d} x
$$

thence (4.37) is fulfilled. Recalling Proposition 4.2, we obtain the desired result.

Remark 4.1. The proof of Theorem 4.3 also characterizes $\theta^{+}$and $\theta^{-}$according to the way $\mathcal{F}$ cuts the abscissa. Indeed, from (4.33), (4.36), (4.39) and (4.41) we obtain

$$
\theta^{+} \in\{\mathcal{F}=0\} \cap\left\{\mathcal{F}^{\prime}>0\right\} \quad \text { and } \quad \theta^{-} \in\{\mathcal{F}=0\} \cap\left\{\mathcal{F}^{\prime}<0\right\} .
$$


Remark 4.2. It would be suggestive to define the function

$$
\mathbb{R} \ni t \longmapsto \mathcal{E}(t):=\int_{\mathbb{R}_{\omega}^{n}} h(x) \gamma_{t}(x) \mathrm{d} x
$$

and to use critical points of $\mathcal{E}$ instead of zeroes of $\mathcal{F}$ in Theorem 4.3.

Analogously, it would be nice to write (4.42) by characterizing $\theta^{ \pm}$in terms of the minimality or maximality attained by $\mathcal{E}$.

Notice that these are only formal statements, since the integral in (4.43) does not converge in general.

The non-degeneracy of an integral function (see [22]) or of its derivative (see [19]) is a classical feature in the construction of chaotic orbits in dynamical systems. In this sense, our functions $\mathcal{E}$ and $\mathcal{F}$ may be seen as Poincaré-Mel'nikov functions.

In dynamical systems, these functions are usually obtained by integrating the perturbation along standard homo/heteroclinics (see, e.g., [3]). In our case, an average on the transversal directions is also needed.

For results and comments on variational non-degeneracy conditions, see [20].

Theorem 4.3 easily gives concrete examples of $h$ 's for which Theorem 2.1 applies:

Corollary 4.1. Let $\kappa>0, \bar{F}$ be an even double-well potential and $F=\kappa \bar{F}$. Given $\omega \in S^{n-1}$, we let

$$
h_{\omega}(t):=\int_{\Omega_{t \omega}} h(z) \mathrm{d} z, \quad \forall t \in \mathbb{R} .
$$

Suppose that $h \in C^{1}\left(\mathbb{R}^{n} / \mathbb{Z}^{n}\right)$ and that

$$
\left\{h_{\omega}=0\right\} \cap\left\{h_{\omega}^{\prime}=0\right\}=\emptyset
$$

Then, there exists $\delta>0$ such that condition $(\mathbf{A})$ holds true for $H=\epsilon h$, provided that $\epsilon \in(0, \delta)$ and $\kappa \geqslant 1 / \delta$.

Proof. If $\bar{\gamma}$ is the heteroclinic of $\bar{F}$, then the heteroclinic of $F$ is

$$
\gamma(x):=\bar{\gamma}(x+(\sqrt{\kappa}-1)\langle\omega, x\rangle \omega) .
$$

Accordingly, from (4.38) we get

$$
\mathcal{F}(t)=\int_{\mathbb{R}_{\omega}^{n}} h\left(y+\left(\frac{1}{\sqrt{\kappa}}-1\right)\langle\omega, y\rangle \omega-t \omega\right) \bar{\gamma}^{\prime}(y) \mathrm{d} y,
$$

and therefore

We now claim that

$$
\mathcal{F}^{\prime}(t)=-\int_{\mathbb{R}_{\omega}^{n}} \partial_{\omega} h\left(y+\left(\frac{1}{\sqrt{\kappa}}-1\right)\langle\omega, y\rangle \omega-t \omega\right) \bar{\gamma}^{\prime}(y) \mathrm{d} y
$$

(4.40) holds if $\kappa$ is large enough.

The proof of (4.47) is by contradiction: if not, by (4.45) and (4.46), there would exist a diverging sequence $\kappa_{j}$ and points $t_{j} \in \mathbb{R}$ for which

$$
\begin{aligned}
0 & =\int_{\mathbb{R}_{\omega}^{n}} h\left(y+\left(\frac{1}{\sqrt{\kappa_{j}}}-1\right)\langle\omega, y\rangle \omega-t_{j} \omega\right) \bar{\gamma}^{\prime}(y) \mathrm{d} y \\
& =\int_{\mathbb{R}_{\omega}^{n}} \partial_{\omega} h\left(y+\left(\frac{1}{\sqrt{\kappa_{j}}}-1\right)\langle\omega, y\rangle \omega-t_{j} \omega\right) \bar{\gamma}^{\prime}(y) \mathrm{d} y
\end{aligned}
$$


Since $\mathcal{F}$ is periodic, say of period $\mathcal{T}$, we may suppose that $t_{j} \in[0, \mathcal{T})$. Hence, there exists $t_{\star} \in[0, \mathcal{T}]$ and a subsequence for which

$$
\lim _{\ell \rightarrow+\infty} t_{j \ell}=t_{\star} .
$$

Therefore, by (4.48) and the Dominated Convergence Theorem,

$$
\begin{aligned}
0 & =\frac{1}{\left|\Omega_{-t_{\star} \omega}\right|} \int_{\Omega_{-t_{\star} \omega}} h(z) \mathrm{d} z \int_{\mathbb{R}_{\omega}^{n}} \bar{\gamma}^{\prime}(y) \mathrm{d} y=\int_{\mathbb{R}_{\omega}^{n}} h\left(y-\langle\omega, y\rangle \omega-t_{\star} \omega\right) \bar{\gamma}^{\prime}(y) \mathrm{d} y \\
& =\int_{\mathbb{R}_{\omega}^{n}} \partial_{\omega} h\left(y-\langle\omega, y\rangle \omega-t_{\star} \omega\right) \bar{\gamma}^{\prime}(y) \mathrm{d} y=\frac{1}{\left|\Omega_{-t_{\star} \omega}\right|} \int_{\Omega_{-t_{\star} \omega}} \partial_{\omega} h(z) \mathrm{d} z \int_{\mathbb{R}_{\omega}^{n}} \bar{\gamma}^{\prime}(y) \mathrm{d} y,
\end{aligned}
$$

that is

$$
-t_{\star} \in\left\{h_{\omega}=0\right\} \cap\left\{h_{\omega}^{\prime}=0\right\} .
$$

This is in contradiction with (4.44) and thus proves (4.47).

Then, the desired claim follows from Theorem 4.3.

As an example, we observe that if, say $\omega=(1,0, \ldots, 0)$, the function

$$
h(x)=\sin \left(2 \pi x_{1}\right)
$$

satisfies the assumptions of Corollary 4.1 and so it gives rise to the multibump solutions of Theorem 2.1.

More generally, when $\omega=p / q$, with $0 \neq p \in \mathbb{Z}^{n}, 0 \neq q \in \mathbb{N}$, a concrete example is given by

$$
h(x)=\sin (2 \pi p \cdot x) .
$$

Also, the function

$$
h(x)=\sum_{i=1}^{N} \sin \left(2 \pi x_{i}\right)
$$

provides an example for any coordinate direction $\omega=(1,0, \ldots, 0),(0,1, \ldots, 0), \ldots,(0,0, \ldots, 1)$.

What is more, given any sequence $\omega^{(j)} \in \mathbb{Z}^{n}$, such that $\omega^{(i)}$ is not parallel to $\omega^{(j)}$ unless $i=j$ (and this may exhaust the rational directions), the function

$$
h(x)=\sum_{k \in \mathbb{N}} \frac{1}{\mathrm{e}^{k}+\left|\omega^{(k)}\right|^{2}} \sin \left(2 \pi \omega^{(k)} \cdot x\right)
$$

satisfies the assumptions of Corollary 4.1 for any $\omega^{(j)}$, since

$$
h_{\omega^{(j)}}(t)=C_{j} \sin \left(2 \pi\left|\omega^{(j)}\right|^{2} t\right)
$$

for some $C_{j}>0$.

\section{REFERENCES}

[1] F. Alessio, L. Jeanjean and P. Montecchiari, Existence of infinitely many stationary layered solutions in $\mathbb{R}^{2}$ for a class of periodic Allen-Cahn equations. Comm. Partial Diff. Eq. 27 (2002) 1537-1574.

[2] S. Allen and J. Cahn, A microscopic theory for antiphase boundary motion and its application to antiphase domain coarsening. Acta Metall. 27 (1979) 1084-1095.

[3] A. Ambrosetti and M. Badiale, Homoclinics: Poincaré-Melnikov type results via a variational approach. Ann. Inst. H. Poincaré Anal. Non Linéaire 15 (1998) 233-252.

[4] D.I. Borisov, On the spectrum of the Schrödinger operator perturbed by a rapidly oscillating potential. J. Math. Sci. (N. Y.) 139 (2006) 6243-6322. 
[5] H. Brezis, Analyse fonctionnelle. Théorie et applications. Collection Mathématiques Appliquées pour la Maîtrise, Masson, Paris (1983).

[6] G. Carbou, Unicité et minimalité des solutions d'une équation de Ginzburg-Landau. Ann. Inst. H. Poincaré Anal. Non Linéaire 12 (1995) 305-318.

[7] R. de la Llave and E. Valdinoci, Multiplicity results for interfaces of Ginzburg-Landau-Allen-Cahn equations in periodic media. Adv. Math. 215 (2007) 379-426.

[8] N. Dirr and E. Orlandi, Sharp-interface limit of a Ginzburg-Landau functional with a random external field. Preprint, http://www.mat.uniroma3.it/users/orlandi/pubb.html (2007).

[9] N. Dirr and N.K. Yip, Pinning and de-pinning phenomena in front propagation in heterogeneous media. Interfaces Free Bound. 8 (2006) 79-109.

[10] N. Dirr, M. Lucia and M. Novaga, $\Gamma$-convergence of the Allen-Cahn energy with an oscillating forcing term. Interfaces Free Bound. 8 (2006) 47-78.

[11] L.C. Evans, Partial differential equations, Graduate Studies in Mathematics 19. American Mathematical Society, Providence, RI (1998).

[12] A. Farina and E. Valdinoci, Geometry of quasiminimal phase transitions. Calc. Var. Partial Differential Equations 33 (2008) 1-35.

[13] G. Gallavotti, The elements of mechanics, Texts and Monographs in Physics. Springer-Verlag, New York (1983). Translated from the Italian.

[14] D. Gilbarg and N.S. Trudinger, Elliptic partial differential equations of second order, Grundlehren der Mathematischen Wissenschaften 224. Springer-Verlag, Berlin, second edition (1983).

[15] V.L. Ginzburg and L.P. Pitaevskiü, On the theory of superfluidity. Soviet Physics. JETP 34 (1958) 858-861 (Ž. Eksper. Teoret. Fiz. 1240-1245).

[16] T. Kato, Perturbation Theory for Linear Operators, Classics in Mathematics. Springer-Verlag, Berlin (1995).

[17] L.D. Landau, Collected papers of L.D. Landau. Edited and with an introduction by D. ter Haar, Second edition, Gordon and Breach Science Publishers, New York (1967).

[18] M. Marx, On the eigenvalues for slowly varying perturbations of a periodic Schrödinger operator. Asymptot. Anal. 48 (2006) 295-357.

[19] V.K. Mel'nikov, On the stability of a center for time-periodic perturbations. Trudy Moskov. Mat. Obšč. 12 (1963) 3-52.

[20] H. Matano and P.H. Rabinowitz, On the necessity of gaps. J. Eur. Math. Soc. (JEMS) 8 (2006) 355-373.

[21] M. Novaga and E. Valdinoci, The geometry of mesoscopic phase transition interfaces. Discrete Contin. Dyn. Syst. 19 (2007) 777-798.

[22] H. Poincaré, Les méthodes nouvelles de la mécanique céleste. Gauthier-Villars, Paris (1892).

[23] P.H. Rabinowitz and E. Stredulinsky, Mixed states for an Allen-Cahn type equation. Comm. Pure Appl. Math. 56 (2003) 1078-1134. Dedicated to the memory of Jürgen K. Moser.

[24] P.H. Rabinowitz and E. Stredulinsky, Mixed states for an Allen-Cahn type equation. II. Calc. Var. Partial Diff. Eq. 21 (2004) 157-207.

[25] J.S. Rowlinson, Translation of J.D. van der Waals' "The thermodynamic theory of capillarity under the hypothesis of a continuous variation of density". J. Statist. Phys. 20 (1979) 197-244.

[26] M. Schatzman, On the stability of the saddle solution of Allen-Cahn's equation. Proc. Roy. Soc. Edinburgh Sect. A $\mathbf{1 2 5}$ (1995) 1241-1275. 